\title{
Adapt! - Agile Project Management Supported by Axiomatic Design
}

\author{
Jakob Weber ${ }^{1, *}$, David Förster ${ }^{1}$, Markus Stäbler $^{1}$ and Kristin Paetzold ${ }^{2}$ \\ ${ }^{1}$ Daimler AG, Process Development and Materials Department, 71059 Sindelfingen, Germany \\ ${ }^{2}$ Universität der Bundeswehr, Institut für Technische Produktentwicklung, 85577 Neubiberg, Germany
}

\begin{abstract}
This paper presents a novel approach for the use of Axiomatic Design Theory in combination with agile project management methods like Scrum for an effective, structured and combined product design and development process. Agile project management methods give a guideline how to manage a project, but there is only minor assistance regarding the actual product development process itself. Axiomatic Design can be used to support these methods in this point. In concrete terms, the results of the decomposition process of this theory can be used to formulate and structure the work packages for the agile project managing process. The Independence Axiom of Axiomatic Design Theory has a substantial contribution by ensuring the independence of the work packages which can be assigned to different project team members and can be processed independently by them. The combination of the different methods not only helps to ensure a good design solution but also helps to work more agile within a project team. The here proposed approach is one part of a holistic product design and development process for changeable production units - called Adapt! - and is described within a use case in the automotive sector.
\end{abstract}

\section{Introduction}

Today's industries and especially the automotive industry face manifold challenges. These challenges exist since the companies are in turbulent settings caused by many different influences like globalization, the introduction of new and disruptive technologies, politics and much more (Figure 1) [1, 2].

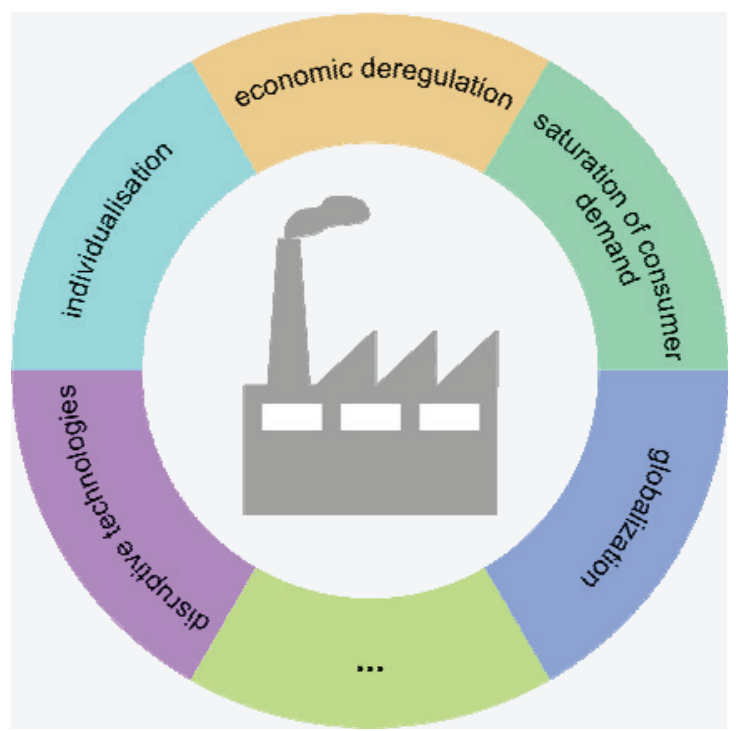

Fig. 1. Turbulences influencing a company.

The turbulences affect the production systems of the companies, which have to react by adapting and changing. This circumstance expresses itself in two different ways. Firstly, the product life cycles of the production units decrease as it is known from the products themselves. In order to be competitive this ends up in the need to accelerate the design and development process of production units.

Secondly, the design of production units gets more and more complex. Caused by new technologies and by the claim of producing an increasing number of variants with the same production unit, the complexity of their design increases.

Major work is done in research according design methods and changeability of production units. Also considerable work is done in context of project management methods in order to give full particulars to a basic concept. However, there is no easy to handle approach combining design methods with project management methods in order to guarantee a holistic design and development process. In this paper, one element of an approach - called Adapt! - is suggested combining the benefits of Axiomatic Design Theory with agile project management methods e.g. Scrum. The benefits of Axiomatic Design are the universal applicability on different disciplines, the focus on product functions and the optimized learnability of this theory. Combining these with the procedure of Scrum ensures a modern technology design process.

As a starting point within this paper related work regarding Axiomatic Design and Scrum is outlined. Based on this, the proposed approach combining Axiomatic Design and Scrum is explained. In the

\footnotetext{
*Corresponding author: jakob.weber@daimler.com
} 
following an example is given, which is envisaged to be processed applying the proposed approach. A discussion on this topic is carried out followed with a conclusion and outlook on future work.

The research questions that have to be answered are the following:

- How can Axiomatic Design support the subsequent project management process after the design phase?

- In what kind of environment is the proposed approach successfully applicable?

- What are the expected benefits of this approach?

\section{Related work}

The following sections describe the fundamentals and the related work of the used methods. The basics of Axiomatic Design Theory are outlined, a brief summary of Scrum as one possible agile project management method is given.

\subsection{Axiomatic Design}

Axiomatic Design is a design theory mainly based on two axioms $[3,4]$. The aim of this theory is to give a scientific and therewith a learnable foundation to design. The theory describes the design process as mapping of requirements and solutions between four domains (see Figure 2). The domains are customer, functional, physical and process domain. Within the customer domain all customer attributes (CA) are summarized. The CA are usually free of any hierarchical order. They will be transformed to functional requirements (FR) within the functional domain. These are all requirements that are demanded of the later product. Each FR will be mapped to a design parameter (DP), which is the physical solution to the specific requirement. The mapping process can be described as switching from the question "what do we want to achieve?" to "how do we achieve it?". By switching repeatedly between these two questions, the design task gets decomposed and more and more specific. This procedure is called "zigzagging". In the next step, the DP of the physical domain are mapped to the process domain by defining suitable process variables (PV). For this work, the relevant step is the mapping from functional to physical domain.

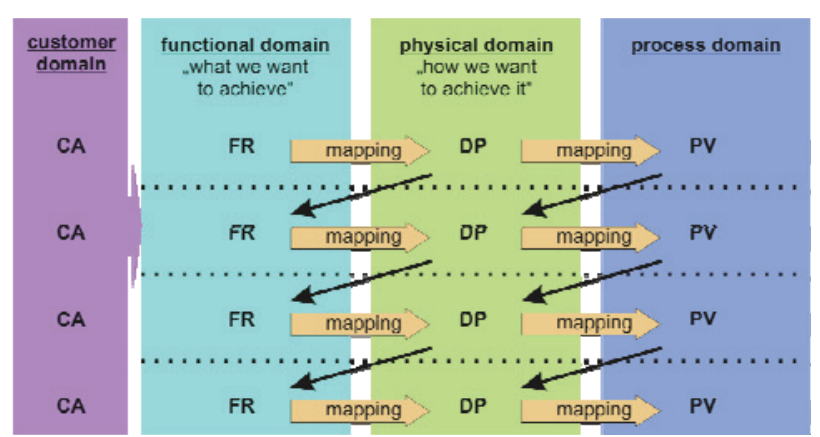

Fig. 2. Decomposition of the design task in Axiomatic Design.
To support the solution determination of the mapping process between the domains, Axiomatic Design uses the already mentioned two axioms. The first axiom Independence Axiom - claims that the FR have to remain independent by finding appropriate DP. The second axiom - Information Axiom - advocates to minimize the information content of the design. This advice is based on the context between information content and the complexity of the proposed solution.

There is major work in the field of production system design. Naming all of them goes beyond the scope of this paper. Rauch et al. give in [5] an overview of publications using Axiomatic Design in this context. One essential conclusion of their work is that almost threequarter of all work focus on the Independence Axiom as it is the case also for this paper. This assumption is also supported by the work of Kulak et al. in [6].

\subsection{Scrum}

Scrum is a framework enabling the treatment of complex projects and problem statements. Furthermore, the creative and productive development of high-graded products is supported. Scrum gives a detailed classification of a project team, strict rules and an accurate course of action. The aim of Scrum is to optimize the work within a project team and to easily control risks by an iterative step by step organization of the development process. Due to Scrum, the process will fulfil the following three characteristics: transparency, inspection and adaption [7].

The main elements of the framework are the Scrum team, the Scrum artefacts and the Scrum events. The Scrum team consists of the Scrum master, the product owner and the development team [7].

The Scrum master is in charge of the abidance of the theory, proceeding and laws of Scrum by the whole team. Furthermore, he tackles all obstacles which hinder the team to fulfil their work.

The product owner is the only person who organizes the product backlog, one of the artefacts of Scrum. This means he prioritizes the product backlog items which have to be developed by the Scrum team. The items of the product backlog are all requirements of the later product and are formulated as clear work orders, which can be fulfilled independently by the development team. Moreover, he is the representative to the stakeholders (e.g. customers).

Other Scrum artefacts are the sprint backlog and the product increment. Related to the product backlog, the sprint backlog is a choice of product backlog items, which should be finished during one sprint. The sprint backlog is being replenished by a plan for delivering the product increment and realizing the sprint goal. The increment is a summary of all accomplished sprint backlog items. At the end of a sprint the new increment must be "done", that means its sprint backlog items must fulfil the definition of done, determined ex ante by the Scrum team. The most important elements of Scrum are the Scrum events, especially the sprint. A sprint is a time box during which the elements of the sprint backlog 
must reach the definition of done. They contain daily meetings of the project team, the daily Scrums, the sprint reviews, termination presentations of the fulfilled tasks at the end of every sprint and the sprint planning. All these elements are a subject to tight restrictions, which must be observed by the team members. The Scrum process is depicted in Figure 3.

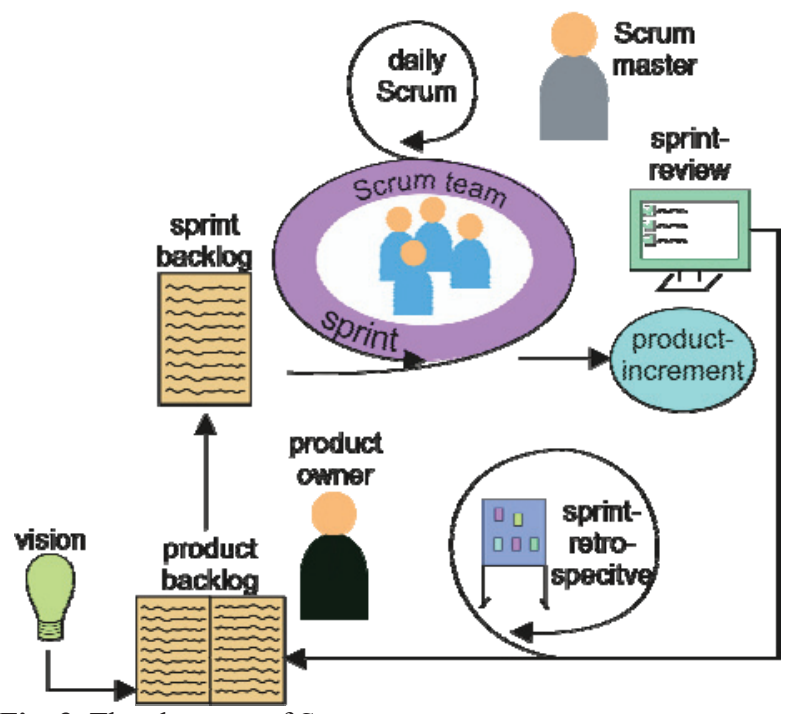

Fig. 3. The elements of Scrum.

\section{Proposed approach}

The development process of innovative production units and in particularly of changeable production units underlies specific challenges. In contrast to a team which develops e.g. software, the development team of changeable production units is usually cross-disciplinary. A common team composition includes engineers and practitioners of different disciplines. So, this interdisciplinary teamwork is one challenge and has to be supported.

Another challenge lies in the nature of work which has to be carried out. The design and development process of production units normally starts with more theoretical and conceptual work and ends with a practical implementation and optimization of the production unit. Hence, there is a switch in the type of work which is depicted in Figure 4. This point makes it inevitable to use a methodology which supports both theoretical and practical work. Design as well as development cannot be treated isolated.

Using Axiomatic Design offers substantial possibilities for lean development processes especially in large organizations. Related work regarding the development of changeable production units is presented in $[8,9]$. To take account of the mentioned shift of theoretical and practical work, these works have to be extended with a procedure to support the practical part of the development work. This is done in a methodology, we call Adapt!. The name refers to the approach itself and also to the product, which is designed and developed with it. Both, the approach and the later product is adaptable to new circumstances.
The proposed procedure is also extended by an analysis and evaluation part, which results in a holistic approach for designing and redesigning changeable production units [10]. The following sections limit on the design and development process of Adapt!.

The basic idea of Adapt! is to provide an easy to understand and learn approach to design. Therefore, the Independence Axiom of Axiomatic Design is used to decompose a design task. This is done in accordance to [9] by only decomposing the task on the highest and most abstract levels. The resulting hierarchical design tree is transformed to a design matrix. It is assumed that the customer attributes of a design task not only result from one customer within a large company, but from different departments. Therefore, it is useful to colorize the resulting functional requirements and design parameters according to the origin of the former customer attribute. So, dependencies in the design matrix are colorized and can be referred to the department which claimed the specific functional requirement. On this basis, it is possible to enter into a dialog with the customer departments and to start a discussion with the aim to overthink some of the stated requirements with the aim of simplifying the design task. After this the decomposition of the design task is done again. In case of obtaining a satisfying design solution according to the Independence Axiom, the result is a conceptual design of a changeable production unit fulfilling the design task [8].

Starting from this interim result, the shift from conceptual to practical work starts. The basic concept has to be refined and a first prototype has to be engineered. For this purpose a development team consisting of several experts of different subjects is initiated. There is also a change of roles of the project manager. From being quite on his own during the design phase, he becomes part of the Scrum team. In the case of using Scrum he becomes product owner (Figure 4).

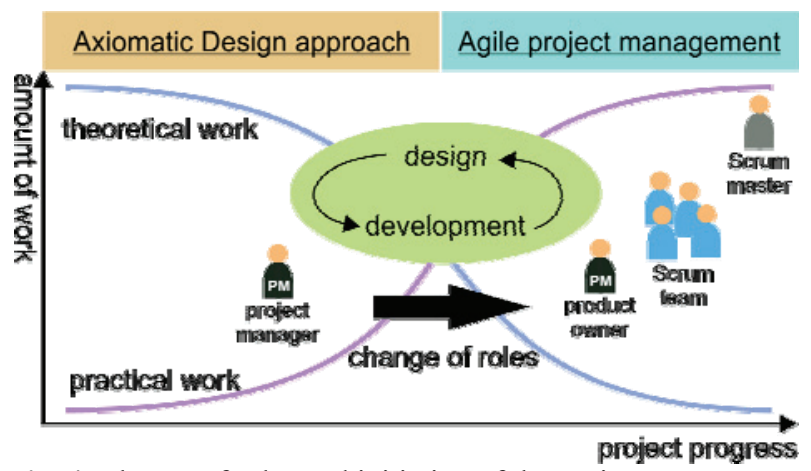

Fig. 4. Change of roles and initiation of the project team.

In order to coordinate their work and to be able to react on unforeseen circumstances, Scrum is a suitable framework as already explained in the related work section. A central aim of Scrum is to enable selforganized work which is done in sprints. In order to describe the work content of each sprint there are so called refinement and sprint meetings. In a refinement meeting the tasks are specified, so that every team 
member knows what to do. In a sprint meeting it is defined which task will be processed during the following sprint.

Within this context, the hierarchical tree of the conceptual design can be used to organize the refinement. This is consistent with the rules of Scrum although they deny any change or exception of the Scrum rules. "The Scrum Team decides how and when refinement is done" [7]. In accordance to this, the presented paper provides a suggestion how the Scrum team can organize the refinement.

The elements of the hierarchical tree of the conceptual design can be seen as rough formulated work tasks for the single Scrum sprints. They can be listed in the product backlog. Some of the elements can already be sufficiently defined to be further processed in a sprint. Others may be too abstract to be already processed. So, they have to be detailed in a refinement meeting. Within this refinement meeting, again, the proposed approach from [9] focusing the Independence Axiom can be used to decompose the design task. If the granularity of the branch of the hierarchical tree is fine enough, they are adequate as a single work task and can be accepted to be consigned to the sprint back log of a future sprint. This is depicted in Figure 5.

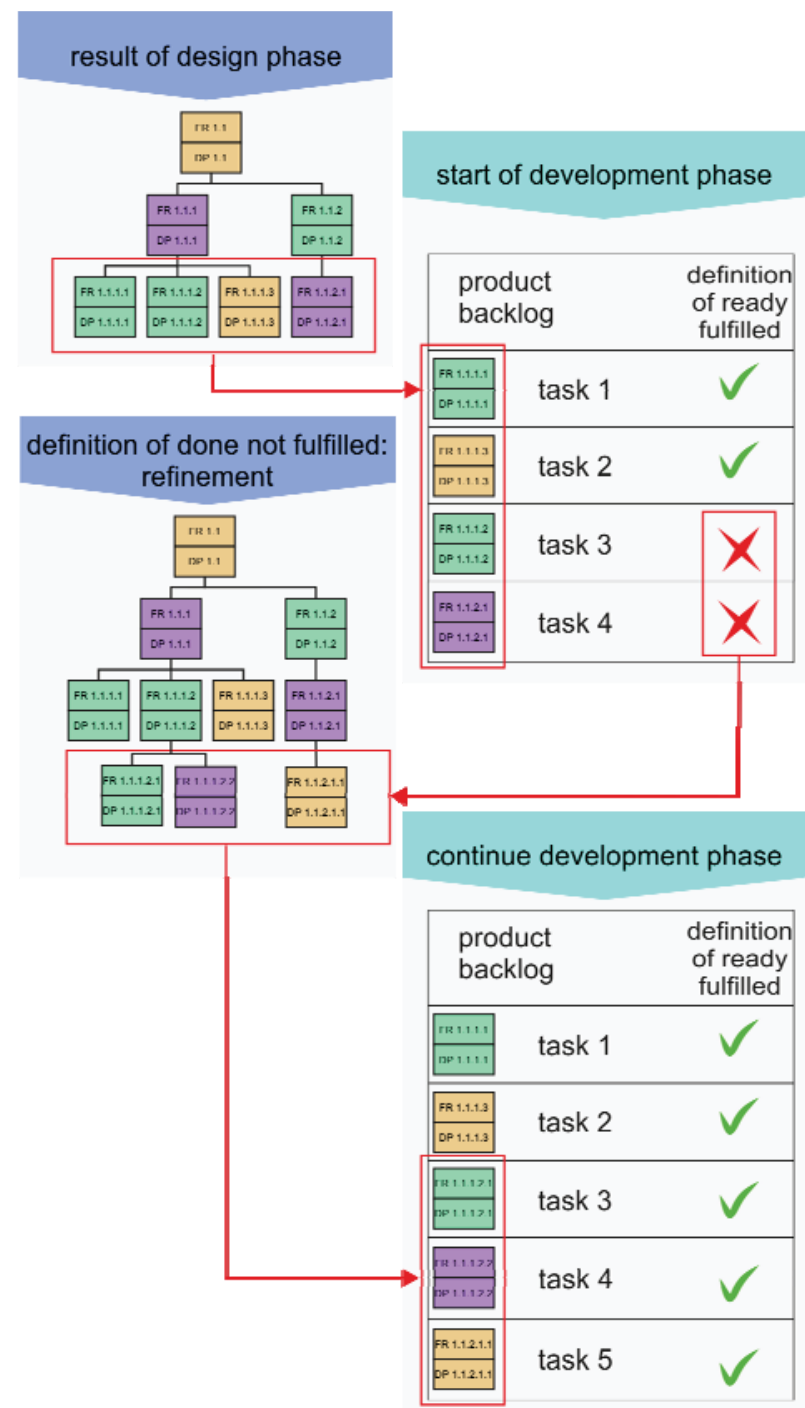

Fig. 5. Supporting Scrum sprint planning with AD.

\section{Practical example}

For illustrating the use of Adapt! hereafter the approach will be explained on a practical example. The described project is in the conceptual phase and Adapt! will be applied from the beginning for the first time. At first the product development process of the upper layers of the hierarchical tree will be illustrated. Subsequently, the distribution of roles by the use of Scrum in an industrial environment will be explained.

In the beginning technical concepts must be worked out. This is done by the project manager who is a development engineer. He summarizes the customer attributes and starts the decomposition of the hierarchical tree. He is doing his work mainly himself. Only in the case of needing further information he asks for bilateral support by experts of the affected department. During this process the elements of the Axiomatic Design tree are colorized according to the department where they originate from. The design parameters are selected by applying the first axiom of Axiomatic Design. The decomposition ends when it is detailed enough to formulate product backlog items out of the functional requirements and related design parameters of the lowest level of the hierarchical tree.

By this point, the Scrum team is initiated with the aim of implementing a mechanical prototype of the later product. In this particular example the team consists of the development engineer, an implementation engineer, a design engineer, a software specialist, an electrician and a mechanic. These specialists have to be deployed to the single functions of the team in a favorable way.

In the development phase the project manager of the design phase should have a leading role in order to have an overview of the project. This is done in the best way when the project manager of the design phase is assigned as product owner of the development phase. By this he keeps the overview of the whole project and can prioritize the work tasks. Furthermore, with his detailed knowledge about the project he can properly communicate with the stake holders.

Another crucial role is given by the Scrum master. For this position the implementation engineer is predestinated. His most important task is to abolish impediments which hinder the Scrum team. In his work as implementation engineer one of his tasks is already the abolishment of impediments during realization phase of a production unit. Hence, he brings the right mind-set to enable the work of the whole Scrum team. The distribution of roles within the Scrum team is illustrated in Figure 6. 


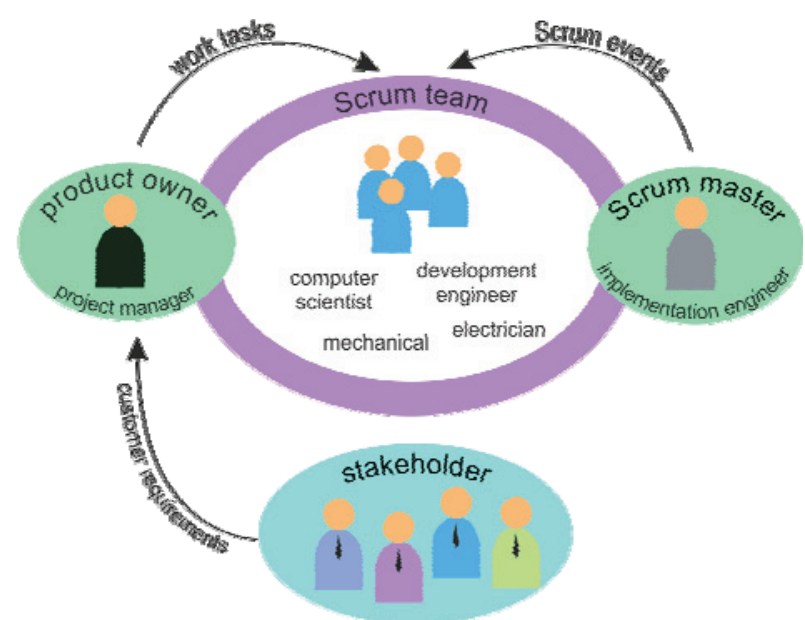

Fig. 6. Distribution of roles within the project team.

With this team composition, the development process starts. This is the current status of the launched project. For the further project's duration, the following benefits are expected:

Through the use of Axiomatic Design Theory, the extensive independence of single work tasks of the later product development process should be ensured. This will be favorable because every team member can work on his own work task independently and does not have to rely on others. This will support an agile project handling and a self-organized way of work. On this basis, a high-capacity utilization of the team members is expectable. So, waiting times are reduced and the design and development process is accelerated.

Finally, the continuous decomposition of the design task leads to an also continuous and complete documentation of the design and development process. This reduces efforts for ex post documenting.

\section{Discussion}

A critical assessment on the proposed approach can be done from the methodical point of view. Two existing and well-established procedures are used. By only combining them, the specific shortcomings of each of the two methodologies remain unchanged. E.g. there is no further work done in supporting creativity during the design phase, which is an often-criticized shortcoming of Axiomatic Design Theory. Furthermore, the execution of Scrum cannot be simplified. Applying Scrum makes it inevitable to have a specific mind-set within the Scrum team and also within the company especially within the management.

However, combining these two procedures helps to innovate our production technologies. To the contrary, by not predetermining every single step and by not overburdening the design process, an easy-to-use approach is achieved.

Another point of criticism might be that there is no strict specification how detailed the Axiomatic Design tree has to be, before initiating the Scrum team.

Quite the contrary, it can be argued, that this is one advantage of Adapt!. The procedure can always be adjusted to the specific needs of a development team.
One more big challenge is the fact, that the members of the Scrum team have different professional qualifications. This is a requirement which originates from the circumstance of developing hardware. According to Scrum every team member is called developer and there is no hierarchy. This might cause troubles due to different qualifications of team members. But the advantages of working in an agile way overtake this challenge.

In comparison to the mentioned disadvantages and challenges there exist also a lot of advantages of this approach. By using the approach, the design and development process is structured. Despite of this structuring the whole procedure is very agile and allows the development team to react on unforeseen events. Overall, it ensures the development of changeable production units.

\section{Conclusion and Outlook}

This paper presented an innovative approach for a consistent product design and development process using Axiomatic Design to support agile project management methods like Scrum.

The need for a consistent approach for design and development of changeable production units was revealed. Moreover, the possibility of using hierarchical trees from the decomposition of the design task with Axiomatic Design as input for the product development process with agile methods like Scrum was shown. In addition to this an example was given, where the proposed approach - a part of a method named Adapt! is applied for the first time. A possible composition of a project team with the aim of designing and developing a changeable production unit was given. The desired benefits of the approach were presented in detail, too. Finally, a critical discussion of the proposed approach was done.

Future work involves the elaboration of the holistic evaluation, design and development process for the design of changeable production units. Furthermore the applicability of the presented approach has to be proven.

\section{References}

1. E. Westkämper, Marktorientiertes Produzieren in dynamischen Strukturen. In: H. J. Warnecke, ed. Fabrikstrukturen im Zeitalter des Wandels welcher Weg führt zum Erfolg? 12-21 (Springer, 1995)

2. H.P. Wiendahl, C.L. Heger, Justifying Changeability. A Methodical Approach to Achieving Cost Effectiveness. Proceedings of CIRP 2nd Int. Conf. on Reconf. Manuf. (2003)

3. N.P. Suh, The Principles of Design (Oxford University Press, 1990)

4. N.P. Suh, Axiomatic Design: Advances and Applications (Oxford University Press, 2001) 
5. E. Rauch, D. T. Matt, P. Dallasega, Application of Axiomatic Design in Manufacturing System Design. A Literature Review, Procedia CIRP 53 1-7 (2016)

6. O. Kulak, S. Cebi, C. Kahraman, Application of Axiomatic Design Principles. A Literature Review. Expert Systems with Applications 37 6705-6717 (2010)

7. K. Schwaber, J. Sutherland, The Scrum Guide (2016)

8. J. Weber, D. Förster, J. Kößler, K. Paetzold, Design of changeable production units within the automotive sector with Axiomatic Design. Procedia CIRP 34 93-97 (2015)

9. J. Weber, J. Kößler, K. Paetzold, An Approach for Industrial Application of Axiomatic Design. Proceedings of ICED (2015)

10. M. Stäbler, J. Weber, K. Paetzold. Implementing Changeability in a System with Prioritization Indicators. Procedia CIRP 44 50-54 (2016) 